\title{
CONSIDERACIONES DOCENTES Y RETOS PARA LA EDUCACIÓN VIRTUAL EN MÉXICO
}

\section{Ramírez León Rodolfo Humberto ${ }^{1}$, Carballo Aguilar Oscar $A^{2} . y$ Álvarez Cisneros Wilder ${ }^{3}$}

\section{Resumen}

El artículo menciona la imperiosa necesidad que tenemos los docentes de afrontar los cambios en el paradigma educativo, que impone la modalidad a distancia. Por lo que inicia describiendo algunos antecedentes de los cambios ocurridos en México durante los inicios del siglo XXI. Incorpora varias de las características de los hogares y usuarios de internet en México, según la Encuesta Nacional sobre Disponibilidad y Uso de Tecnologías de la Información en los Hogares 2015 (ENDUTIH). Aborda además, algunas concepciones teóricas de autores que conciben la educación virtual como parte de la Sociedad del Conocimiento, dando a conocer algunas de las características que tienen los alumnos del nuevo esquema virtual y concluye destacando los retos que tienen las Instituciones para incorporar educación formal y continua, aprovechando los modernos recursos tecnológicos.

Palabras clave: Educación en línea, TIC aplicadas a la educación, cambio paradigma educativo.

\section{Abstract}

The article mentions the imperious need that the teachers have to face the changes in the educational paradigm, which imposes the distance modality. So begins by describing some background of the changes that occurred in Mexico during the beginning of the 21st century. It incorporates several of the characteristics of households and Internet users in Mexico, according to the 2015 National Survey on Availability and Use of Information Technology in Homes (ENDUTIH). It also addresses some theoretical conceptions of authors who conceive of virtual education as part of the Knowledge Society, revealing some of the characteristics that the students have of the new virtual scheme and concludes by highlighting the challenges that the Institutions have to incorporate formal education and Continuous, taking advantage of the modern technological resources.

\footnotetext{
${ }^{1}$ Universidad Autónoma de Chiapas, México.

${ }^{2}$ Universidad Autónoma de Chiapas, México.

${ }^{3}$ Universidad Autónoma de Chiapas, México.
}

\begin{tabular}{c|c|c|c}
\hline Rev. Ciências Humanas & Frederico Westphalen, RS & Pg. $27-40$ & Set./dez. 2018 \\
\hline \multicolumn{2}{c}{ Recebido em: 04/01/2019 } & Aceito em: 04/02/2019 \\
\hline
\end{tabular}




\section{(1)URI|}

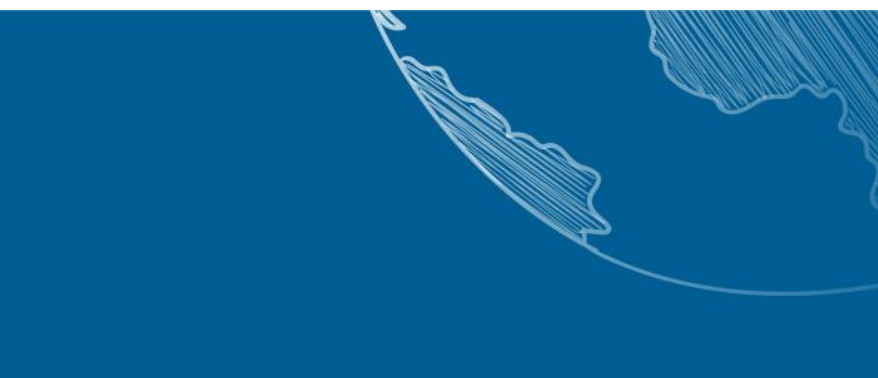

KEYWORDS: Online education, ICT applied to education, educational paradigm shift.

\section{INTRODUCCIÓN.}

Atrevernos a hacer las cosas diferentes en la educación, es sin duda el reto más importante que tenemos los docentes en el Siglo XXI, dentro de la denominada Sociedad de la información y el Conocimiento. Para Julio Cabero: "no es suficiente con poseer la capacidad de memorizar la información, sino que se requiere la capacidad de reformular la realidad, aportar soluciones a los problemas, ser creativo e innovador en la aplicación de las soluciones a los problemas, saber moverse en un contexto cercano y futuro incierto y dinámico, y utilizar enfoques holísticos para saber desenvolverse en sistemas tan complejos y dinámicos como los que se nos presentan.” Cabero (2015).

Además, no debemos olvidarnos que en lugar de pensar en tecnologías futuras, lo que debemos hacer es invertir esfuerzos en construir modelos de enseñanza para obtener el máximo partido a las tecnologías que tenemos actualmente en nuestros centros educativos, ya que la innovación no se consigue por la novedad de aplicación tecnológica, sino por la aplicación de criterios para conseguir nuevos escenarios formativos y comunicativos.

Por ello, la incursión de las Instituciones educativas en el ámbito virtual o a distancia, prevé grandes retos, uno de ellos, la osadía de hacer las cosas diferentes, pues repetir la clase tradicional no es lo más adecuado. Veamos algunos de las características más relevantes de este tema.

\section{ANTECEDENTES EN MEXICO DE LA EDUCACIÓN VIRTUAL}

Durante el año 2006, se desarrollaba en México una serie de esfuerzos académicos con el fin de sentar bases sólidas para realizar, de manera conjunta, un

\begin{tabular}{c|c|c|c|}
\hline Rev. Ciências Humanas & Frederico Westphalen, RS & Pg. $27-40$ & Set./dez. 2018 \\
\hline Recebido em: 04/01/2019 & Aceito em: 04/02/2019 \\
\hline
\end{tabular}




\section{CIÊNCIAS HUMANAS}

REVISTA DO PROGRAMA DE PÓS-GRADUAĞ̈O EM EDUCAĞ̄o

\section{(1) URI|}

sistema de educación a distancia en México. Para tal logro, el entonces Secretario

General de la ANUIES mencionaba varios factores importantes:

1. Que el aula, profesores o Instituciones no son los únicos depositarios del conocimiento, sino que ahora éste se produce y construye en otros ambientes, como la casa, centros de trabajo, y más recientemente, a través de las modernas redes virtuales.

2. La enorme necesidad data también de la velocidad vertiginosa con que se suceden los avances científicos, lo que requiere de una educación permanente.

3. La posibilidad que da la educación virtual es puesta de manifiesto, al mencionar que se interseccionan tres puntos importantes: las posibilidades, la tecnología y la pedagogía. Castañares (2006).

Desde entonces, se destacaba el enorme incremento de la educación virtual, a partir del año 2007, llegando a ocupar un $65 \%$ de la oferta de cursos a nivel mundial, mientras que en Latinoamérica se creaban Instituciones para encabezar los esfuerzos sobre el uso y explotación del esquema tecnológico como serían: el Centro de Alta Tecnología a Distancia (UNAM), el proyecto e-México, entre otros.

La iniciativa e-Mexico articula los esfuerzos de entidades y dependencias públicas, operadores de redes de telecomunicaciones, cámaras y asociaciones vinculadas con las tecnologías de la información y la comunicación con el propósito de ampliar diversos servicios. Este sistema, tal como lo dice en su portal (www.emexico.gob.mx) tiene como objetivo: "eliminar las barreras que actualmente existen para obtener información y servicios públicos. Busca también reducir las brechas tecnológicas al interior del país y entre la población mexicana con el resto del mundo". El sistema es una red de conexión satelital y por Internet que enlaza puntos de conectividad de alta velocidad a través de los Centros Comunitarios Digitales, localizados en escuelas, bibliotecas, centros de salud, oficinas de correos y edificios de gobierno de los municipios. Según datos de e-México, se encuentran instalados 7,200 


\section{CIÊNCIAS HUMANAS}

REVISTA DO PROGRAMA DE PÓS-GRADUAĞ̈O EM EDUCAĞ̄o

\section{(1) URI|}

Centros en todo el país. Un aspecto que debe destacarse es que éstos permiten el acceso a la conectividad a las personas que por cuestiones económicas no pueden hacerlo desde sus casas.

Castañares también destaca que llama la atención sobre lo que podría suceder a futuro. La empresa Merril Lynch \& Co., dedicada al análisis económico, afirma que para el año 2025 habrá 160 millones de personas realizando estudios de educación superior, y que de ellas 40 millones estarán vinculadas con la educación en línea en algún momento de su vida, esto es, una de cada cuatro, lo cual nos muestra el impacto que tendrán los procesos de virtualización en la educación terciaria (Merril Lynch, 2000).

Respecto al sector empresarial la situación también es significativa, puesto que el uso de las TIC ha tenido un amplio auge y aplicación para apoyar diversos procesos de capacitación, no sólo a nivel mundial, puesto que también en México se ha desarrollado bastante. A manera de ejemplo tenemos los siguientes datos: Entre los años 2001 y 2005, como lo cita el portal tendenciasdigitales.com, el número de empleados con acceso a la red desde sus oficinas, aumentó de 100 a 409 millones, en todo el mundo. Mientras que, en América Latina, también se avanza con celeridad en la incorporación de esquemas tecnológicos en la empresa. Y en México, existe un alto porcentaje de las empresas que utilizan formación en línea lo realizan debido a la calidad de la capacitación, así lo manifestó 90.42 por ciento de ellas, en tanto que 58.6 por ciento afirma que se vio beneficiado en materia de productividad y éxito comercial tras la implementación de los cursos virtuales.

En el ámbito educativo se tienen dos problemas importantes que atender, uno de ellos se refiere a que los programas que se tienen en el posgrado y la educación continua, donde, al menos en México, $60 \%$ de los programas ofrecidos se ubicaban en el nivel de posgrado, siendo en las especializaciones los programas mayormente ofrecidos; y en segundo lugar los de maestría. Las áreas de mayor cobertura, al igual que las presenciales, son las de las Ciencias Sociales y Administrativas, seguidas por las de educación y humanidades y, siguiendo este orden decreciente, se ofertan programas 


\section{CIÊNCIAS HUMANAS}

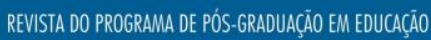

\section{(1) URI|}

en ciencias de la salud, ingeniería y tecnología, ciencias agropecuarias, y en último lugar los de ciencias naturales y exactas.

Finalmente, Castañares indica que existen varios retos a superar, para permitir al sistema virtual emerger con fuerza, a saber:

a) Se tiene que ampliar la oferta educativa virtual. Es claro que muchas instituciones deben reforzar su infraestructura tecnológica, de tal manera que les permita ofrecer programas de esta naturaleza.

b) Otra situación que se presenta en el contexto de la educación superior virtual en México se refiere al poco impacto que tienen los programas ofrecidos por las instituciones de educación superior. Se ofrecen cursos aislados, y más encaminados hacia la educación continua, cuando existen rezagos en otras áreas del conocimiento que también deben atenderse. En consecuencia, lo que se requiere es diversificar los campos de conocimiento, crear nuevas carreras, incorporar innovaciones tecnológicas para la formación de los nuevos profesionales y asegurar su inserción en los mercados laborales.

c) También debemos contar con una amplia planta de docentes, administrativos y directivos que requieren formarse en el uso de los medios. Si bien existen diversos cursos de esta naturaleza, es importante resaltar que en la mayoría de los casos se han dejado de lado a los cuerpos directivos y de gestión, actores importantes para el adecuado desarrollo de los programas educativos.

d) Es importante romper resistencias y crear una cultura de uso de la tecnología en apoyo a los procesos educativos. Cuando hablamos de una cultura queremos decir que no basta con cursos de formación y capacitación que se centren exclusivamente en su aplicación, también se requiere que los docentes desarrollen habilidades pedagógicas y diseñen contenidos y nuevas estrategias de enseñanza y de aprendizaje, como se llevan a cabo en algunas instituciones educativas, pues los procesos de virtualización no son el paso mecánico de contenidos a diferentes medios. 


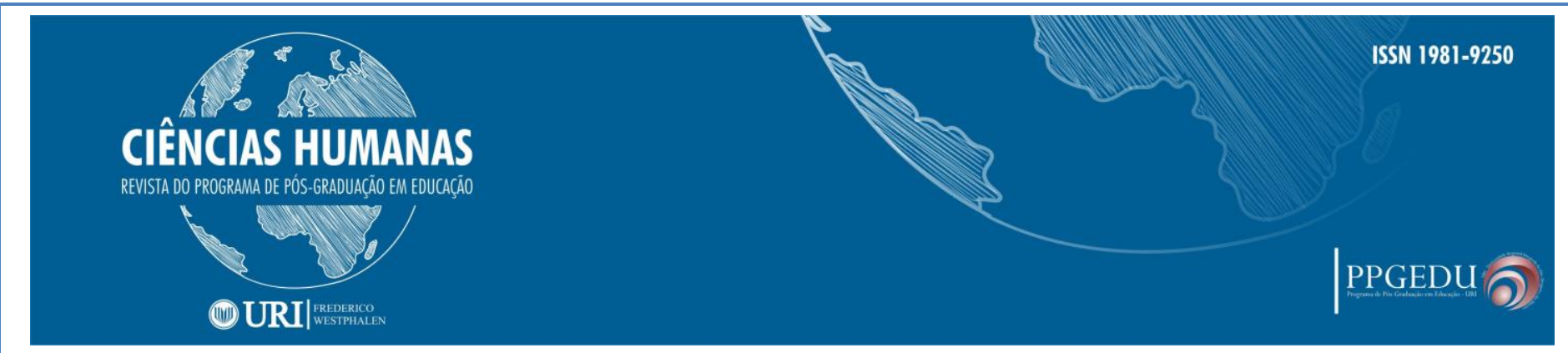

e) Un aspecto, estrechamente vinculado con lo anterior, y que sin embargo no es exclusivo de propuestas virtuales, se refiere a la importante necesidad de construir modelos educativos que efectivamente estén centrados en el aprendizaje. Hoy los requerimientos de la sociedad así lo demandan, pues el estudiante es responsable de su proceso de formación.

f) Otra cuestión que no puede soslayarse se refiere a los presupuestos. Éstos son limitados para la educación superior en su conjunto, y prácticamente nulos para modalidades alternativas a la presencial.

g) Así como, la falta de un marco regulatorio que dé certeza jurídica, como es en el caso de los sistemas presenciales, es otro de los retos a los que tenemos que hacer frente. Se hace indispensable en la medida en que se incrementa el número de instancias que ofertan programas bajo este modelo.

\section{USUARIOS DE INTERNET E INFRAESTRUCTURA EN HOGARES DE MÉXICO}

Con base en la Encuesta Nacional sobre Disponibilidad y Uso de Tecnologías de la Información en los Hogares (ENDUTIH) que se levantó en 2017, se hacen destacar los siguientes rubros:

$\checkmark$ En México hay 71.3 millones de usuarios de Internet, que representan el 63.9 por ciento de la población de seis años o más. El 50.8\% son mujeres y el 49.2\% son hombres. El aumento total de usuarios respecto a 2016 fue de 4.4 puntos porcentuales.

$\checkmark$ El grupo de población con mayor proporción de usuarios de internet es el de los hombres de 18 a 34 años de edad, casi el $85 \%$ de la población de este grupo usa este servicio, mientras que el grupo de edad con menor uso son las mujeres de

\begin{tabular}{c|c|c|c}
\hline Rev. Ciências Humanas & Frederico Westphalen, RS & Pg. 27 - 40 & Set./dez. 2018 \\
\hline \multicolumn{2}{c}{ Recebido em: 04/01/2019 } & Aceito em: 04/02/2019 \\
\hline
\end{tabular}




\section{CIÊNCIAS HUMANAS}

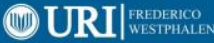

55 años y más, lo cual indica que el uso de Internet es predominante en la población joven del país.

$\checkmark$ Las principales actividades de los usuarios de Internet en 2017, son: obtener información (96.9\%), entretenimiento (91.4\%), comunicación (90.0\%), acceso a contenidos audiovisuales $(78.1 \%)$ y acceso a redes sociales (76.6 por ciento).

$\checkmark$ Ya sea mediante conexión fija o móvil, 17.4 millones de hogares disponen de Internet (50.9 por ciento del total nacional), lo que significa un incremento de $3.9 \%$ respecto al año anterior, por lo que en menos de 10 años, los hogares con conexión a Internet se duplicaron.

$\checkmark$ El análisis geográfico muestra que el uso del internet es un fenómeno urbano, puesto que el $86 \%$ de los usuarios de este servicio se concentran en estas zonas.

En el análisis detallado por rangos de edad se pueden observar diferencias notables. Mientras que poco más de la mitad (53.9\%) de los niños de entre 6 y 11 años señaló utilizar Internet con cierta regularidad, entre los adolescentes de 12 a 17 años la proporción alcanza el $85.9 \%$, proporción semejante a la observada para individuos de entre 18 y 24 años (83.1\%). Incluso para el siguiente grupo de edad (25 a 34 años) la proporción se mantiene por encima de dos de cada tres (71.1\%). INEGI (2017).

Otros puntos relevantes que arroja ENUDITH se refiere a que durante el año 2017, el $72.2 \%$ de la población de seis años o más utiliza el teléfono celular. Ocho de cada diez, disponen de celular inteligente, con lo cual tienen la posibilidad de conectarse a Internet. El número total de usuarios que sólo disponen de un celular inteligente creció de 60.6 millones de personas a 64.7 millones de 2016 a 2017.

Así podemos mencionar las Tecnologías de la Información y las Comunicaciones (TIC) constituyen herramientas privilegiadas para el desarrollo de los individuos y de las sociedades al facilitar el manejo de información: crearla, compartirla, modificarla, enriquecerla y transformarla en conocimiento. El uso de las TIC se encuentra cada vez más difundido, tanto por la aparición de nuevos dispositivos como por la reducción de los costos asociados. Por lo que se constituye como un área de 


\section{CIÊNCIAS HUMANAS}

REVISTA DO PROGRAMA DE PÓS-GRADUAĞ̈O EM EDUCAĢĀO

\section{(1) URI|}

oportunidad, que la Escuela debe de aprovechar para ampliar su cobertura, sobre todo en la Educación Superior.

\section{TEORIA Y DEFINICIÓN DE LA EDUCACIÓN VIRTUAL}

Se ha identificado que quienes deseen incursionar en este reto, atraviesan una mayor dificultad cognoscitiva que los tradicionales sistemas presenciales, entre ellos se encuentran: Desarrollo de competencias académicas y habilidades de pensamiento analítico, analógico, crítico, dialógico y hermenéutico, y de actitudes de trabajo colaborativo o de valores como el respeto a la propiedad intelectual. Romero (2007).

Así Romero menciona que "... lo virtual consiste en el uso intensivo del ciberespacio, y prácticamente nada de interacción cara a cara, dada la facilidad para acceder a información hipertextual y la comunicación en línea, en tiempo real", y además que están presentes el uso de multimedios como serían el correo electrónico \{elearning\}, el ambiente hipertextual que provee la web \{on-line learning o aprendizaje distribuido \} y más recientemente, los sistemas basados en tecnología móvil: e-books, PC de bolsillo, palm books, y teléfonos celulares $\{$ m-learning\}, entre otros adelantos tecnológicos. Derivado de estas notas características, la educación virtual permite el incremento del número de alumnos dispersos a lo largo y ancho de diversos países; obliga a las instituciones educativas a disponer de complejos equipos de procesamiento de información y de telecomunicaciones; igualmente les permite reducir la infraestructura escolar tradicional, como el número de aulas, pero a la vez las obliga a transformar dicha infraestructura en espacios dialógicos y de acceso a la información, lo que conlleva costos más altos.

En 2011, García propone una nueva teoría para la educación virtual denominada diálogo didáctico mediado, basando su propuesta en la comunicación a través de los medios, formulando las bases de la citada teoría, iniciando con algo característico: debe existir una comunicación mediada entre docentes y alumnos, estableciendo un diálogo, donde el estudiante no se limite a responder, sino a preguntar o a iniciar el mismo. Un elemento muy importante a considerar es la tutoría, pues se constituye en algo 


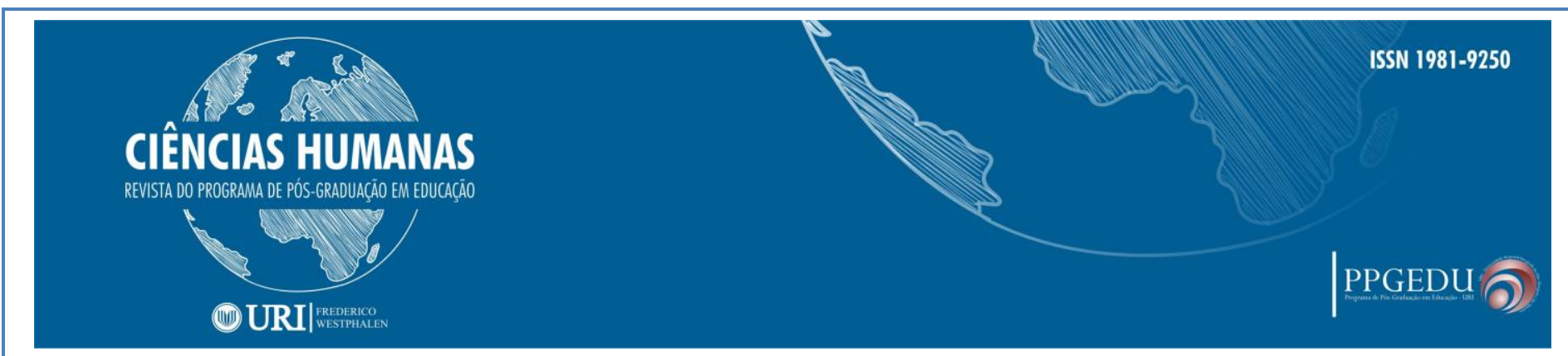

sustancial y singular de los sistemas a distancia, el tutor suele ser el rostro, la imagen de esta forma de enseñar-aprender al establecer un sentimiento de relación personal entre el que enseña y el que aprende. La teoría propuesta se considera como integradora y comprensiva de los modelos teóricos existentes como el de industrialización, teoría de la independencia, el de distancia transaccional, entre otros. Se divide el esquema en cuatro grandes cuadrantes, donde interactúan los aspectos docentes, pedagógicos, de aprendizaje y los medios. García (2011).

\section{EL ALUMNO EN AMBIENTES VIRTUALES.}

Debido a lo incipiente de la incursión en este esquema se dice que conocer al estudiante virtual constituye un requisito que contribuye a generar ambientes de formación de mayor pertinencia y sentido que respondan a la gran diversidad de necesidades educativas en Latinoamérica, por lo que se justifica desde una perspectiva social, pues en la medida en que identifiquemos las implicaciones pedagógicas resultantes del conocimiento de este estudiante, se facilitará la tarea de convocar y retener a quienes buscan la formación virtual como una alternativa. Así, podemos pensar en que las tecnologías se constituyan en un factor real de acceso e inclusión tanto en el sistema educativo como en diversas experiencias informales de aprendizaje. Desde esa perspectiva, por la supuesta posibilidad de conectividad en cualquier lugar de la geografía, se podría afirmar que se contribuye de manera real a la disminución de la brecha tecnológica. Parra de Marroquín, O. (2008)

Parra destaca que, según investigaciones realizadas en USA, los jóvenes participan de manera natural en cursos virtuales, llegando a un 65\% de jóvenes menores de 18 años inscritos en este tipo de cursos, en Latinoamérica aún no se tiene este dato, pero sería interesante conocerlo. Además, resume algunas características de los estudiantes virtuales, a saber:

- Personas automotivadas, ya sea por circunstancias personales o porque poseen altos niveles de motivación intrínseca, que no requieren mucho de otros para mantenerse en el aprendizaje. 


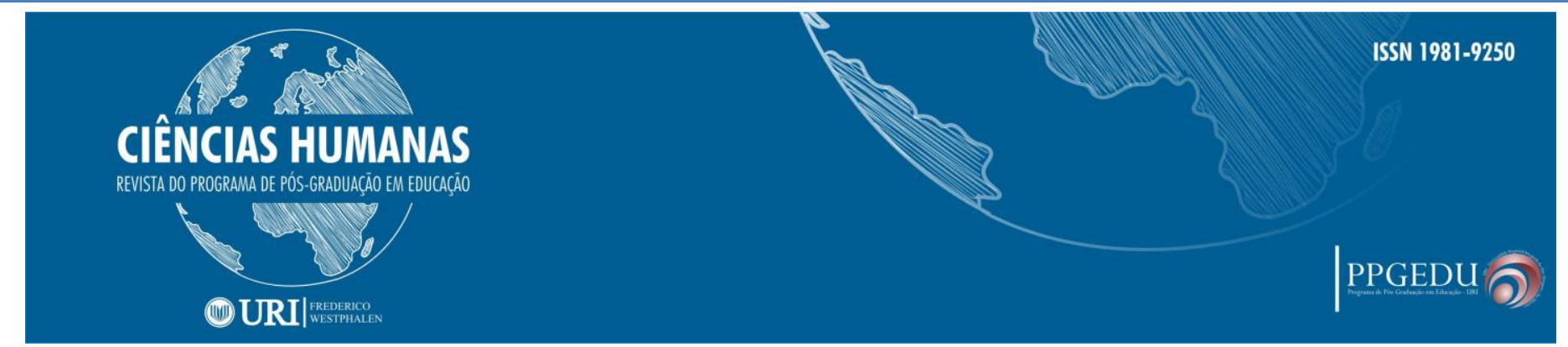

- Personas autodisciplinadas que logran manejar los cambios motivacionales y, a pesar del desánimo que pueda surgir en momentos específicos, son capaces de continuar.

- Personas tecnológicamente hábiles que comprenden fácilmente el funcionamiento de las herramientas.

- Personas con buena capacidad para comunicarse por escrito.

- Personas que asumen en serio los compromisos, especialmente cuando un curso requiere buena cantidad de tiempo y energía.

- Personas que creen en la posibilidad de aprender de diversas maneras que trascienden el aula de clase.

Menciona que en muchas ocasiones la habilidad tecnológica no siempre está presente, y puede terminar un curso persistiendo debilidades en el manejo de la tecnología. Por lo que quizá sería más correcto referirse a una actitud de aceptación de la tecnología, como condición que genera facilidad para involucrarse con el curso.

\section{RETOS PARA LOS SISTEMAS VIRTUALES.}

El autor Parra de Marroquín menciona los siguientes retos para los sistemas virtuales:

Primer reto: La diferencia entre forma y fondo. La diferencia entre forma y fondo, tan valiosa para nuestro pensamiento habitual ha sufrido confusiones desde que asumimos que el medio es el mensaje, lo que ahora podemos traducir como que la tecnología en ella misma.

Segundo reto: Ya no al diseño pedagógico sino epistemológico. Para continuar el acercamiento al tema Manuel Cebrian de la Serna afirma que "el poder en el aula ya no está en el control de la información, sino, más bien, en la capacidad de construir el conocimiento o en la capacidad de permitir crear un verdadero autoaprendizaje tutorizado en nuestros estudiantes: la información ya no es conocimiento"

\begin{tabular}{c|c|c|c}
\hline Rev. Ciências Humanas & Frederico Westphalen, RS & Pg. $27-40$ & Set./dez. 2018 \\
\hline \multicolumn{2}{c|}{ Recebido em: 04/01/2019 } & Aceito em: 04/02/2019 \\
\hline
\end{tabular}




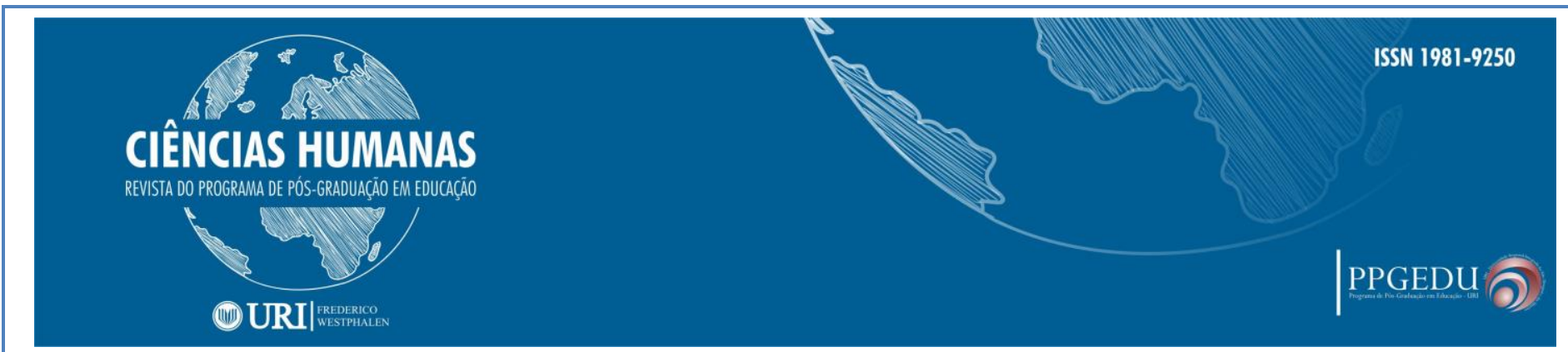

Tercer reto: La tecnología como contenido del pensamiento. Los estudiantes de sistemas de educación virtual deben poseer conocimientos y habilidades preliminares en el orden del manejo de la tecnología de comunicación y procesamiento de información.

Cuarto reto: el tránsito de la descripción informativa a la hermenéutica de los datos y de los hechos. Los hechos, los datos de la historia, los textos y los comportamientos cotidianos son en ellos mismos, silentes, dado que no vienen con manuales explicativos ni con aproximaciones a su significado o sentido.

Quinto reto: el aprendizaje colaborativo y dialógico en cursos masivos. El trabajo colaborativo constituye una de las premisas de los cursos en línea, lo que es, de inicio, paradójico, en la medida que cada uno de los estudiantes efectúa su propia gestión formativa desde el aislamiento de su oficina o de su estudio; la mayoría de los estudiantes prefiere los cursos presenciales justamente por la realimentación directa, inmediata y en tiempo real; por el contrario, en los cursos en línea la realimentación entre estudiantes y la realimentación que proviene del profesor, se efectúan en tiempos diversos, si bien dentro de márgenes muy delimitados.

Sexto reto: el aislamiento del aprendizaje. Es necesario recordar que destaca un inevitable sentimiento de aislamiento y soledad que se manifiesta en la urgencia de encontrar la inmediata respuesta del profesor o del tutor ante sus dudas, preguntas y aportaciones, como si el profesor mantuviera su presencia continua en el otro lado de la línea.

Séptimo reto: Ruptura y conversión cognoscitiva. Asumimos que todo aprendizaje autogestivo es indudablemente, una conversión precedida de una ruptura cognoscitiva. No nos referimos a las rupturas epistemológicas de la historia en las que los paradigmas imperantes dejaron de ofrecer explicaciones a los temas y problemas humanos del momento, y por ello generaron cosmovisiones radicalmente diferentes.

Octavo reto: Las competencias sociales y humanas. El modelo de cursos en línea asume como parte importante el trabajo colaborativo, como ya se ha dicho, pero asume también la formación de competencias de carácter social y humano que más difícilmente pueden alcanzarse. La dificultad se explica porque dichas competencias deben partir de una sensibilización cognoscitiva ante los problemas sociales pero deben

\begin{tabular}{c|c|c|c}
\hline Rev. Ciências Humanas & Frederico Westphalen, RS & Pg. 27 - 40 & Set./dez. 2018 \\
\hline Recebido em: 04/01/2019 & Aceito em: 04/02/2019 \\
\hline
\end{tabular}




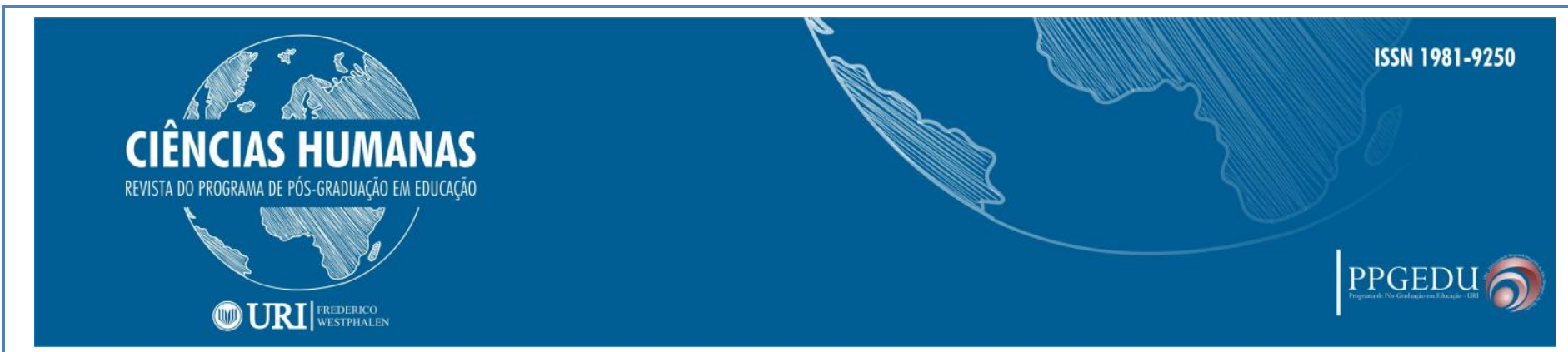

aterrizarse en gestiones específicas, en las que se noten las evidencias de su logro. Ante esta circunstancia importan las preguntas por la posibilidad de la humanización y formación ciudadana y la del desarrollo de habilidades para la realización profesional y la felicidad cotidiana.

Noveno reto: Educación virtual y TIC para la dignidad y la vida humanas. Seguiremos viviendo por mucho tiempo en el contexto de la educación virtual, el desarrollo de competencias y la sociedad del conocimiento, pero por ello mismo el siguiente reto que nuestra cavilación propone es el referente humano de toda TIC y de todo aprendizaje virtual. De suyo la preocupación de todo modelo educativo es el hombre y la colectividad y en ello muchos coincidimos, como el filósofo alemán Keiner.

Décimo reto: El mito de la ligereza de los cursos y los riesgos de deshonestidad académica. Dada la distancia real entre el profesor y los estudiantes, lo mismo que la distancia entre estudiantes, ubicados en diversas ciudades y países, no hay manera de identificar personalmente el aprovechamiento específico de cada uno. Tampoco existe una manera de identificar que el alumno efectúe sus trabajos y no un amigo, como solía ocurrir en la secundaria. No es difícil reconocer trabajos que no son sino documentos bajados de alguna página de Internet e incluso existen mecanismos para saberlo.

Undécimo reto: La carga simbólica de los cursos en línea y de la educación. A juzgar por su dimensión tecnológica, los cursos en línea poseen una alta carga simbólica, si bien, dentro de los márgenes del formalismo lógico, matemático e informático. Mediante iconos, password y códigos comprendemos y accedemos a las computadoras, a los servidores de Internet, a los programas y a todos los comandos. El buen estudiante de cursos en línea debe ser un experto en la semiótica de las máquinas.

\section{CONCLUSIONES}

Es necesario considerar la perspectiva de Parra, quien manifiesta "ante la euforia de la educación en línea, con todas sus bondades y ventajas, quedan retos cuya dimensión apenas se perfila: los que van desde el dominio de las TIC hasta la construcción epistemológica del conocimiento, pasando por la humanización del aprendizaje sustentado en el instrumental tecnológico"

\begin{tabular}{c|c|c|c}
\hline Rev. Ciências Humanas & Frederico Westphalen, RS & Pg. $27-40$ & Set./dez. 2018 \\
\hline Recebido em: 04/01/2019 & Aceito em: 04/02/2019 \\
\hline
\end{tabular}




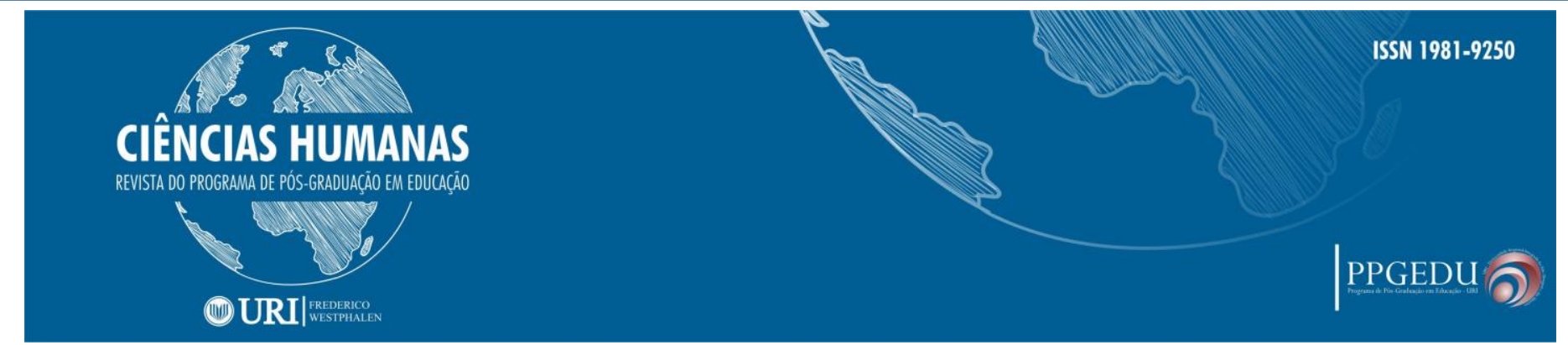

Por ello, es fundamental que el profesor desarrolle material electrónico adaptado a la asignatura y lo ponga a disposición del alumno en una plataforma de aprendizaje electrónico efectiva. Por otro lado, la implantación de un curso a distancia en la enseñanza superior da también mayor importancia al trabajo personal del alumno. Todo esto hace que hoy día sea imprescindible la aplicación de una metodología docente basada en el aprendizaje por descubrimiento del alumno cuyo principio elemental es que lo que el alumno puede hacer por sí mismo, no lo debe hacer el profesor. Esto significa que el alumno trabaja de forma independiente con la mínima intervención del profesor y alcanza los objetivos del curso descubriendo por sí mismo aquello que tiene que aprender. Este tipo de aprendizaje se consigue con el desarrollo de guías de trabajo autónomo (Camacho-Pérez, 2009) que refuerzan la gestión del autoaprendizaje y la responsabilidad del alumno, respetando al mismo tiempo las distintas capacidades, disponibilidades horarias, necesidades y ritmos de aprendizaje de los alumnos.

\section{BIBLIOGRAFÍA}

Aguilera, Escabias, et al. Importancia de las guías de trabajo autónomo en la educación virtual. Experiencias en el aprendizaje online de Estadística aplicada con Moodle. Revista Investigación operacional. Vol. 32, No. 2, Granada, España.

CABERO ALMENARA J. 2015. Reflexiones educativas sobre las tecnologías de la información y la comunicación (TIC). CEF, núm. 1 (mayo-agosto 2015, págs. 19-27)

Castañares, R. L. (2006). Hacia un sistema virtual para la educación en México, 3. ANUIES. Apertura. Año 6 No. 3.

García, Lorenzo. (2011). Perspectivas teóricas de la educación a distancia y virtual. Revista Española de Pedagogía, año LXIX, no, 249, 255-272 


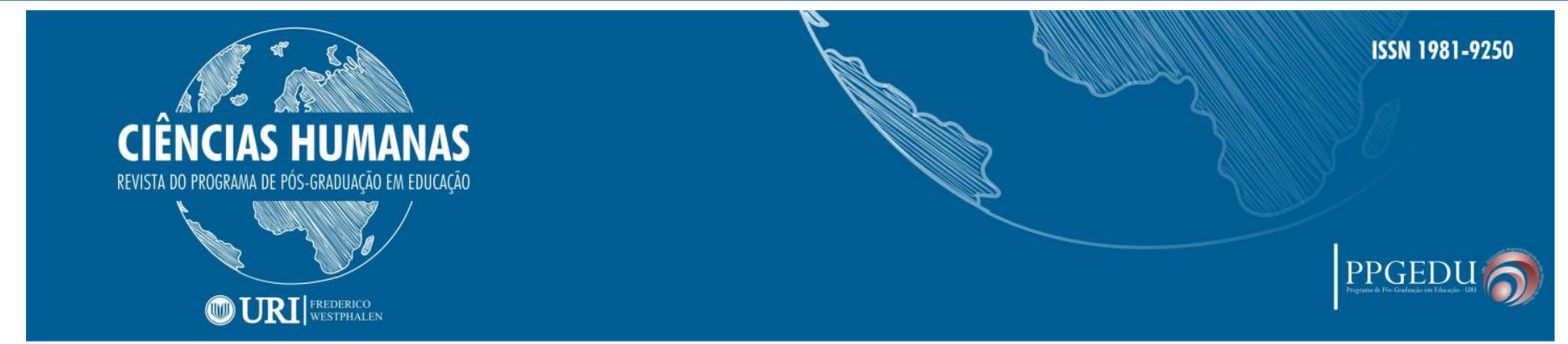

INEGI. Encuesta Nacional sobre Disponibilidad y Uso de TIC en Hogares (ENDUTIH). México. 2017.

Parra de Marroquín, O. (2008). El estudiante adulto en la era digital. (Spanish). The adult student in the digital era. (English), 8(8), 35-50

Romero Romett Miguel. Retos Cognitivos de la Educación Virtual. Acercamiento al proceso de aprendizaje de los cursos en línea. Filososofía. Pag. 216-233 